\title{
What is the likely future of real-time transient stability ?
}

\author{
Damien Ernst ${ }^{*}$, Louis Wehenkel ${ }^{\dagger}$ and Mania Pavella $\ddagger$ \\ * University of Liège, Belgium, Email: dernst@ulg.ac.be \\ $\dagger$ University of Liège, Belgium, Email: 1.wehenkel@ulg.ac.be \\ $\ddagger$ University of Liège, Email: mania.pavella@ulg.ac.be
}

\begin{abstract}
Despite very intensive research efforts in the field of transient stability during the last five decades, the large majority of the derived techniques have hardly moved from the research laboratories to the industrial world and, as a matter of fact, the very large majority of today's control centers do not make use of any real-time transient stability software. On the other hand, along all these years the techniques developed for realtime transient stability have mainly focused on the definition of stability margins and speeding-up techniques rather than on preventive or emergency control strategies. In the light of the above observations, this paper attempts to explain the reasons for lack of industrial interest in real-time transient stability, and also to examine an even more fundamental question, namely: is transient stability, as has been stated many decades ago, still the relevant issue in the context of the new power systems morphology towards more dispersed generation, higher penetration of power electronics, larger and more complex structures, and, in addition, of economic and environmental constraints? Or, maybe, there is a need for techniques different from those developed so far ?
\end{abstract}

\section{INTRODUCTION}

Since the late sixties, when the first control centers where put in operation, there has been a sustained research effort aiming to develop methods for real-time transient stability. While these methods have evolved over the years, their main focus has steadily been to define appropriate stability margins and to speed-up computation so as to comply with real-time constraints, and much less to address preventive or emergency control strategies.

On the other hand, despite very intensive research effort in this field world-wide, the large majority of the derived tools have hardly moved from the research laboratories to the industrial world and, as a matter of fact, the very large majority of today's control centers does not make use of any real-time transient stability software.

Several hypotheses about this lack of industrial success of real-time transient stability can be proposed. The first that comes to mind is the mild interest that system operators have for real-time transient stability tools. Indeed, it seems that around the world, power systems tend to be relatively immune to transient instability and, if they are not, power system engineers have managed to design - often by relying on timedomain simulation software and simple trial and error methods - some well-working strategies (these strategies are of diverse nature but can usually be assimilated to operational guidelines or special protection schemes) to ensure a safe operation of their system. Another explanation for this lack of success is that to migrate to the industry, the new tools produced by researchers should at least offer significant advantages over simple time-domain simulation software used in a 'clever way'. Often the advantages put forward were in terms of computational performances, but at the price of accuracy. It may be that the researchers have given too much value to computational savings - possibly due to the fact that they have not anticipated well enough the tremendous decrease of the cost of computing power over the years. To this awkwardness to trade accuracy for computer savings is also added the fact that researchers in transient stability have mostly preferred to develop analysis tools, rather than control tools which, however, could be more interesting to power system operators.

While it is difficult to predict what is going to be the future of real-time transient stability - especially since with the changing structure of the electrical power systems (more power electronics, distributed generation, etc.) the root causes behind the loss of synchronism phenomena may soon appear different from those of the past - it is important that power system researchers avoid redoing mistakes of the past. We suggest that this could be achieved if every researcher in the field of transient stability defines his project while keeping in mind the following two questions: "Does transient stability still matter ?" and, if yes, "What does the industry really expect in terms of transient stability assessment and control tools ?"

This paper aims to encourage researchers to question current practices and past research efforts, as well as to investigate the intrinsic assumptions behind the approaches that have been developed for transient stability and control over the past 30 years, so as to see whether these latter approaches still hold under the perspective of current and future evolutions of electric energy systems.

\section{RECALL OF THE CURRENT CONTEXT}

Power system security has always been essentially and intrinsically in conflict with economic and, more recently, with environmental requirements. Power systems security control indeed aims at making decisions in various time horizons so as to prevent the power system operation from internally undesired situations, and in particular to avoid large catastrophic outages, such as blackouts and brownouts. To do this implies to sacrifice to some extent economy and ecology; the main problem is to arbitrate among these three criteria (security, 
economy, ecology). Traditionally, security control has been divided in two main strategies: preventive and emergency control. In preventive security control, the objective is to prepare the system when it is still in normal operation, so as to make it able to face future (uncertain) events in a satisfactory way. In emergency control, the disturbing events have already occurred, and thus the objective becomes to control the dynamics of the system in real-time in such a way that consequences are minimized, in spite of what has already happened to it.

Preventive and emergency controls differ in many respects, among which we list the following three [4], [5]:

1) Types of control actions: generation rescheduling, network switching reactive compensation, sometimes load curtailment for preventive control; direct or indirect load shedding, generation shedding, shunt capacitor or reactor switching, network splitting for emergency control.

2) Uncertainty: in preventive control, the state of the system is well known but disturbances are uncertain; in emergency control, the disturbance has already happened and may have been identified in real-time, but the exact state of the system is difficult to measure in real-time and hence often only partially known; in both cases, dynamic behavior is uncertain because many details about the system components are ignored and because the intrinsic complexity of the system leaves many doors open for unexpected failures and abnormal behaviors.

3) Open versus closed loop: preventive control is generally of the open loop feed-forward type; emergency control may be closed loop, and hence more robust with respect to uncertainties. In the past, many utilities have relied on preventive control in order to maintain system security at an acceptable level. In other words, while there are many emergency control schemes installed in reality, the objective has been to prevent these schemes as much as possible from operating, by imposing rather high objectives to preventive security control. As to any rule, there are exceptions: for example controlled generation shedding has been used more or less extensively in North America to handle transient stability problems; in the same way, corrective control has been used in many systems as an alternative to preventive control in the context of thermal overload mitigation.

Nowadays, where the pressure is to increase trading and competition and to reduce environmental impact in the power system field, preventive security control is being considered more and more as an impediment with respect to these other objectives in competition with security; in turn, this breeds strong incentives to resort less on preventive control and more often on emergency control, but poses also the question of how to design future power systems so as to meet their essential objectives (security, economy and ecology) over the medium term (ten or twenty years) or the longer term.

The disappointment and positive motivation behind this paper are essentially the following. First, we observe that despite significant research on transient stability during about 40 years (a rather long period), notable progress in system theory and information technology (not to say), and significant investments from industry to support these efforts (despite restructuring), not much has changed in the everyday practice, be it in preventive or in emergency mode of control. Second, we believe that it is worth to re-discuss the question of whether transient stability is indeed still a problem of paramount practical importance, at least in the way it has been posed over the last 50 years, or more positively, whether it should be posed in a different way.

\section{STATE OF THE ART IN TRANSIENT STABILITY ASSESSMENT AND CONTROL RESEARCH}

The current state of the art has been mostly driven by the need for methods appropriate for rapid contingency assessment. In the race, competition has been between time-domain simulation and so-called direct methods. The latter, based essentially on Lyapunov-like approaches, are intrinsically less demanding from the computational point of view, but their application in power systems transient stability is linked to simplifying assumptions; on the other hand, they are potentially providing richer information (for control and sensitivity analyses; e. g., see [1], [3]). The time-domain methods, on the other hand, have been taking advantage of computational progress and synergy with other simulation domains, which led them to become the "state of the art" in the field; but time domain simulation is not providing directly guidelines for control and sensitivity analyses. Nevertheless, these simulation and direct approaches strongly rely on the validation of their models, which in the context of power system transient stability may in most cases not be ascertained. More recently, hybrid time-domain direct methods have been proposed, able to relax direct methods from simplifying assumptions, to combine the functionalities of both, time-domain and direct methods, and, further, to extend them [2].

The need for designing fast enough methods for contingency assessment, while real several years ago, is not anymore a valuable motivation for research. Costs of computers, and technology for easy parallel computations, make it more or less straightforward to carry out transient stability assessment (even for large scale systems) in real-time with existing time-domain or direct methods. Thus it is up to the industry to determine whether these solutions are effective from an economic point of view.

Hence, the open question for the research community concerns the development of preventive and emergency control strategies, able to face potential problems of transient instability. This turns out to be a much more difficult issue, we believe open for further research. In this context, hybrid methods provide today a partial answer, although maybe too partial for practical use. Indeed, these methods can determine generation rescheduling (active power), but reactive power management and power system topology have not yet been addressed in a satisfactory way. 
Possible directions for future research concern the application of optimal control theory, model predictive control, and more generally optimization-based formulations of the control problem. Some of these directions have already been proposed in the research community, but they have not yet been considered as serious alternatives by the industry.

More fundamentally, we raise the question of whether the transient stability problem, as it has been posed many decades ago, is still a relevant issue, in particular if we take into account the evolution of power systems towards more dispersed generation and higher penetration of power electronics and other kinds of FACTS devices.

\section{AN OUTLOoK ABOUt the FUtURE OF TRANSIENT STABILITY AND CONTROL}

Power systems are evolving towards more and more dispersed generation (co-generation, photovoltaic power, wind energy, others) that will be connected at low or medium voltage levels. At the same time, the geographical scale of interconnection is widening. This means that the power system is becoming a more an increasingly huge and complex system, interconnecting a much larger number of generators than in the past.

Extending current practices of transient stability to these future systems would essentially mean that an interconnection is safe only if no contingency is liable to lead to loss of synchronism even of the smallest interconnected generator or at the remotest end of the interconnection.
The question we want to raise is whether the proper criterion for exploiting such future systems is indeed the constraint that no individual (or small group) of generators loses synchronism or whether it would be more appropriate to define a system wide criterion that would in particular take into account the propagation of the loss of stability.

Some arguments show that these two points of view cannot be aligned.

\section{ACKNOWLEDGMENTS}

Damien Ernst acknowledges the financial support of the Belgian National Fund of Scientific Research (FNRS) of which he is a Research Associate.

\section{REFERENCES}

[1] M. A. Pai. Energy Function Analysis for Power System Stability. Power Electronics and Power Systems. Kluwer Academic Publishers, 1989.

[2] M. Pavella, D. Ernst, and D. Ruiz-Vega. Transient Stability of Power System. A Unified Approach to Assessment and Control. Power Electronics and Power Systems. Kluwer Academic Publishers, 2000.

[3] M. Pavella and P.G. Murthy. Transient Stability of Power Systems: Theory and Practice. John Wiley \& Sons, 1994.

[4] L. Wehenkel. Emergency control and its strategies. In Proceedings of the 13-th PSCC, pages 35-48, Trondheim, Norway, 1999.

[5] L. Wehenkel, D. Ruiz-Vega, D. Ernst, and M. Pavella. Real Time Stability in Power Systems - Techniques for Early Detection of the Risk of Blackout, chapter Preventive and emergency control of power systems, pages 199232. Kluwer Academic Publishers, 2005. 